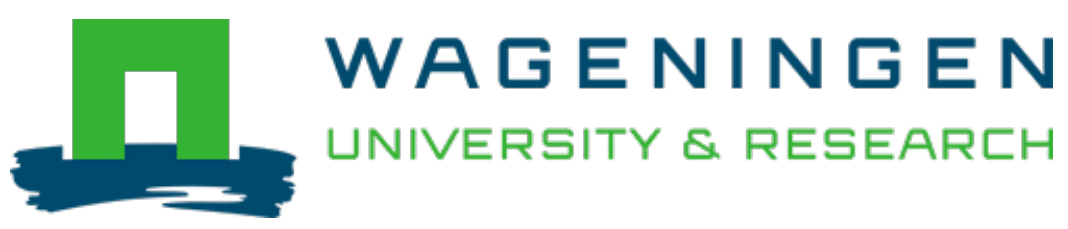

\title{
Outlook - Challenges and Perspectives for Management of Diseases Caused by Pectobacterium and Dickeya Species
}

Plant Diseases Caused by Dickeya and Pectobacterium Species

Gijsegem, Frédérique; Toth, Ian K.; Wolf, J.M.

https://doi.org/10.1007/978-3-030-61459-1_9

This article is made publicly available in the institutional repository of Wageningen University and Research, under the terms of article $25 \mathrm{fa}$ of the Dutch Copyright Act, also known as the Amendment Taverne. This has been done with explicit consent by the author.

Article 25 fa states that the author of a short scientific work funded either wholly or partially by Dutch public funds is entitled to make that work publicly available for no consideration following a reasonable period of time after the work was first published, provided that clear reference is made to the source of the first publication of the work.

This publication is distributed under The Association of Universities in the Netherlands (VSNU) 'Article $25 \mathrm{fa}$ implementation' project. In this project research outputs of researchers employed by Dutch Universities that comply with the legal requirements of Article $25 \mathrm{fa}$ of the Dutch Copyright Act are distributed online and free of cost or other barriers in institutional repositories. Research outputs are distributed six months after their first online publication in the original published version and with proper attribution to the source of the original publication.

You are permitted to download and use the publication for personal purposes. All rights remain with the author(s) and / or copyright owner(s) of this work. Any use of the publication or parts of it other than authorised under article $25 \mathrm{fa}$ of the Dutch Copyright act is prohibited. Wageningen University \& Research and the author(s) of this publication shall not be held responsible or liable for any damages resulting from your (re)use of this publication.

For questions regarding the public availability of this article please contact openscience.library@,wur.nl 


\title{
Chapter 9 \\ Outlook-Challenges and Perspectives for Management of Diseases Caused by Pectobacterium and Dickeya Species
}

\author{
Frédérique Van Gijsegem, Ian K. Toth, and Jan M. van der Wolf
}

\begin{abstract}
The soft rot Pectobacteriaceae (SRP) cause diseases of significant economic impact on a wide range of crop and ornamental plants. By their nature these pathogens have a broad host range, a wide climatic tolerance and can be found in a variety of environmental niches (with knowledge of the latter likely to expand greatly in the coming years). While these pathogens can be devastating, they can also be very subtle, living on the surface of plants as part of their normal life cycle or remaining dormant within plants in a latent state until suitable environmental conditions trigger disease development. This can make control difficult and finding ways to control these diseases has been a major challenge. However, new methods are on the horizon in the form of greatly improved diagnostics, biocontrol and other integrated pest management (IPM) methods and the development of new resistant varieties through the use of exciting new technologies. This chapter summarises the major characteristics of SRP and looks to the future and their ultimate control.
\end{abstract}

In this book we have presented both historical and more recent advances in the study of the soft rot Pectobacteriaceae (SRP) genera Dickeya and Pectobacterium, which are responsible for wilting and soft rot in many crops, flowers and ornamentals worldwide. However, despite numerous studies on the taxonomy, epidemiology, environmental niches, plant hosts and geographical distribution of these pathogens, we still have some way to go in the development of effective management strategies. In this chapter we outline the complexity of the disease problem we encounter and give a perspective on improved management options for the future.

F. Van Gijsegem ( $\varangle)$

Institute for Ecology and Environmental Sciences, Sorbonne Université, INRAE, 75005 Paris,

France

e-mail: vangijse@agroparistech.fr

I. K. Toth

James Hutton Institute, Dundee, UK

J. M. van der Wolf

Wageningen University, Wageningen, The Netherlands 


\subsection{Challenges in the Control of Soft Rot Pectobacteriaceae}

\subsubsection{Global Spread}

SRP are spread globally, being present ubiquitously on all continents (with the possible exception of Antarctica). While the worldwide distribution of Pectobacterium spp. has been known for some years disease-causing Dickeya spp., which were previously described as being present mainly in tropical and subtropical regions, also appear to be expanding their global distribution. Indeed Dickeya spp. cause disease in potato as far north as Finland near the Arctic Circle and as far south as New Zealand. All SRP species do not, however, have the same geographic distribution and the occurrence of different species may largely differ depending on climate, trading policies and hosts. Climate dependency is mainly relevant to $P$. atrosepticum, which prefers cooler humid temperate regions, unlike other SRP that can cause disease in a wider range of climates. The role that trade in plant material has on the spread of these pathogens is evident. For example, D. solani, which has not yet been reported in North America, Africa and Oceania (Table 8.1), emerged about 20 years ago in Europe and has since spread throughout much of Europe. Isolates from these different regions are highly clonal, providing good evidence that the pathogen was spread from a single initial infection via the seed trade. D. solani is not present on potato in Scotland due to very strict quarantine restrictions imposed on the movement of traded potatoes to this country. For other species, the role of trade is less obvious, e.g. $P$. brasiliense isolates cluster at the DNA level according to their geographical origins (Sect. 5.5). Easily accessible improved molecular tools now provide an opportunity for us to better understand the routes and spread by the different SRP spp. around the world.

\subsubsection{Broad Host Range}

In addition to geographical distribution, management of SRP is affected by the broad host range of most SRP species, which includes both monocot and dicot plants, comprising vegetables, cereals, ornamentals and woody plants (such as pear and apple trees), with the list of hosts continuing to increase. Most Dickeya spp. cause diseases in many plants, except $D$. paradisiaca which has only been isolated from banana. Species within the genus Pectobacterium show considerable variation in the number and diversity of plant species that can act as a host (Table 3.1), e.g. P. carotovorum and $P$. versatile have broad host ranges, although $P$. atrosepticum appears to be specific to one (potato) or few hosts. Also, some Pectobacterium isolates have been isolated from monocots, where they appear to be more pathogenic than on dicots, e.g. P. zantedeschiae (Sect. 2.3). For other species, which have been characterized only recently, e.g. P. polaris, $P$. punjabiense, P. parmentieri and P. parvum, knowledge about their spread in different hosts or environments is limited (Table 3.1). 


\subsubsection{Population Structure}

Management of SRP is hampered by rapid changes in population structures. This has been particularly clear in potato in Europe, where $P$. atrosepticum was historically the main disease agent. The 1970s saw the first occurrences of $D$. dianthicola infections and in the 2000s a major outbreak of blackleg disease caused by D. solani spread rapidly across the continent and into Israel. Yet since 2014 D. solani infections have become marginalized to be replaced by major occurrences of $P$. brasiliense and $P$. parmentieri. While $D$. solani on potato probably arose through a host shift from ornamentals (possibly imported) to potato, $P$. brasiliense and $P$. parmentieri appear to have been present in diseased crops for decades and the reasons for these abrupt occurrences/re-emergences are currently not clear. One possible explanation is that improved detection of $D$. solani and the efficient discard of contaminated seed lots has imposed a selective pressure against $D$. solani, thus giving less competitive isolates the chance to compete. Another explanation is that, as with $D$. solani, different species may arrive via trade in other plants into Europe (since potato seed is not imported) where they are then able to jump hosts, establish and spread. Furthermore, the high variability present in some SRP may hinder the efficiency of detection assays. For example, this was the case in a recent US outbreak in potato, where the responsible strain was found to carry a deletion in the gene targeted by the PCR assay. Examples of where strain variation can have an effect include the use of primers derived from the hrp genes, which encode the Type III secretion system, since some species lack this secretion system (Table 4.2), while primers derived from the pectate lyase encoding gene cluster pelADE, used for detecting the Dickeya genus, do not recognize $D$. paradisiaca. This therefore highlights the risk of basing SRP detection on only one set of primers. In addition, these diagnostic methods do not account for the differences in virulence between isolates of the same species, even if otherwise highly related genetically. This is the case for $D$. solani, which includes isolates that are almost clonal and yet contains both highly aggressive and avirulent strains.

\subsubsection{Environmental Diversity}

SRP present in the environment around agricultural and horticultural crops play an important role in the initial infection of those crops and can be responsible for the introduction of new aggressive variants responsible for disease outbreaks. Bacterial soft rot is essentially a disease affecting agricultural or horticultural crops. Nevertheless, SRP have been isolated from asymptomatic weeds, including those grown in open fields or present near surface waters used for irrigation (Sect. 3.2.4). SRP are also found in several other environments including water, air, soil, insects and other invertebrates (See Sect. 3.3). This includes areas remote from arable land, including winter mountain snow, waterfalls, rainwater, rivers, well- and ground-water, the seas and oceans, indicating the potential for long distance dissemination of these species 
(Sect. 3.3.1). Extensive characterisation of SRP populations in surface waters has recently allowed the identification of several new species where, so far, no related isolates have been found on plants or in international bacterial collections (Table 3.1). The latter may be due to the fact that few isolates from water have been collected in the past and so there is a big gap in our knowledge of these species and the extent of the environmental niches they occupy. Strains of Dickeya spp. from such environments possess the same arsenal of virulence genes as those isolated from infected plants and no obvious habitat-specific traits have been identified (Chap. 4). The reasons why these species have not been isolated from plants is not clear, but it may simply be a matter of looking in the wrong place. It can also not be ruled out that with changing cropping systems and climate these species may become a threat.

Continued efforts have been made to discover the main source(s) of contamination by SRP. While we know that the main route of infection and dissemination is via latently contaminated plant material, produced locally or moved over large distances through trade, other sources cannot be ignored. These sources are responsible for initial and ongoing contamination of plants and lead to dissemination and disease in later generations, and in certain circumstances will play a role in the movement of a pathogen between hosts, e.g. the emergence of $D$. solani in Europe is thought to have arisen by the transfer of the pathogen from ornamentals to potato and, similarly, the recent emergence of $D$. dianthicola in potato in Australia may have moved from dahlia grown on land subsequently containing infected potato (Chaps. 3 and 7).

\subsubsection{Latent Infection}

Latent infection, where the pathogen is present in the plant in the absence of symptoms, is another important consideration as it makes it increasingly difficult to control spread. Identifying the major source(s) of primary infections has still not been fully achieved, especially for axenically grown seed stocks, to allow improved procedures to be developed to help avoid such latent infection. The level of contamination/latent infection detected via laboratory testing often does not correlate with the incidence of blackleg observed the following year in the field, mainly due to the strong influence of certain environmental conditions increasing the shift from latency to disease expression. Thus, even with the use of modern diagnostics, the associated costs of testing should be considered to determine the (economic) value to growers of providing diagnostic information. For these reasons the implementation of routine detection in a commercial environment has not, so far, be used to any great extent. 


\subsubsection{Crop Protection Chemicals and Monogenetic Resistance}

Unlike for many crop diseases, crop protection chemicals have not been available for diseases caused by SRP, mainly due to the relatively low economic value of global crop losses caused by these pathogens compared to others such as late blight but also the close taxonomic status of SRP to human and animal gut flora, i.e. chemicals that disrupt SRP could also have a detrimental effect on human health. The main approach for combating any plant disease is the use of varieties that are resistant or more tolerant to the corresponding pathogens. Unfortunately, there is no evidence of gene-for-gene resistance against SRP in any studied plant. In potato, quantitative traits useful to confer resistance have been found in wild Solanum spp. but no genes have so far been isolated (Sect. 6.6). The search for resistance is further hindered by the tetraploid structure of the genome of commercial crops such as potato. This absence of control chemicals and lack of resistance are the major limitations for controlling SRP diseases. Efforts are ongoing from both academic and industry representatives in the hope for a breakthrough, at least in the case of resistance.

\subsection{New Perspectives for Disease Control}

Given the lack of control chemicals and resistant varieties, controlling diseases caused by SRP have relied on certification, good storage and hygiene. However, recent advances in several areas, but particularly in molecular biology and genomic technologies, have resulted in new avenues for potential control, which are briefly outlined below.

\subsubsection{Improved Taxonomy and Diagnostics}

A thorough knowledge of the diversity of the target pathogen is indispensable in a management strategy. Thanks to the advent of whole-genome sequencing (WGS), the classification and naming of SRP has been greatly improved in recent years. Reevaluation of their taxonomy has led to the current description of 19 Pectobacterium and 12 Dickeya species (Sects. 2.2 and 2.3). This effort, at least for Dickeya, has enabled every strain present in international culture collections analysed so far to be placed into one of the described species (Sect. 2.3). WGS also allows rapid recognition of new species. It can be expected that further studies, especially on isolates from habitats such as marine waters, soil and insects, will reveal yet more species. Detailed genetic analyses of such species support identification of the pathogen(s) involved in an outbreak and its subsequent tracking. For example, the separation of the highly ubiquitous $P$. carotovorum into several species, including those recently described $(P$. 
parmentieri, $P$. polaris, $P$. versatile, $P$. peruviense and $P$. parvum) showing different aggressiveness and host specificities, will help to support risk assessment across a range of crops.

WGS has also allowed the development of reliable diagnostics using nucleic acid-based amplification methods (Sect. 5.3). Specific PCR primers have now been developed for most SRP species, especially for those causing blackleg and soft rot diseases of potato (Sect. 5.3 and Table 5.1). The development of more targeted diagnostics will help to generate data on the prevalence of specific SRP clades, in turn helping to assess the impact of infections on yield loss and the cost effectiveness of management strategies. New developments in diagnostics will allow efficient surveys of unexplored bacterial communities that may act as reservoirs for new pathogenic strains. For example, the expansion of SRP host range to trees was recently described for D. fangzhongdai and D. dadantii in fruit trees (Table 3.1) and a putative Pectobacterium sp. causing wetwood disease in several forest and garden trees in Russia affecting 30-40\% of birch trees in some Siberian areas (Sect. 7.2.5). Similarly, we still know little about putative vectors of SRP, such as insects and invertebrates, especially those in direct contact with plant roots (Sect. 3.3.3) or those SRP living in association with weeds and ornamentals. Using improved diagnostics to gain a better understanding of these potential vectors it may, in turn, be possible to develop vector-specific diagnostics to add to our capacity for predicting disease outbreaks.

\subsubsection{Disease Resistance}

While no gene-for-gene resistances have been identified against the SRP, it is hoped that the increasing accessibility of genomic data, together with our enhanced molecular knowledge of disease resistance, may lead to further resistance gene discovery. Combined with new effective genetic tools, such as CRISPR/CAS 9 technology, this may then lead us to identify genes useful for resistance/tolerance that can ultimately be used in potato and other commercially important crops. There is clearly more that could be done using biotechnology as new genes and resistance-related mechanisms are discovered and this offers the most likely route to resistance. However, the lack of social and political acceptability is a major obstacle in the release of such crops (Sect. 6.6.5). An additional opportunity comes from the recent development of inbred diploid potato lines, which overcome the limitations of breeding with tetraploid lines, and will help to accelerate the breeding process should suitable candidate resistance genes or markers become available.

\subsubsection{Biocontrol}

New possibilities for disease control are being investigated through efforts to find biocontrol agents able to limit the spread of SRP. These include various antagonist 
bacteria and bacteriophages (Sect. 6.5). Some promising results in glasshouses and limited field assays have been reported but more work is needed to develop them into commercial products that can manage disease, especially given the diversity of SRP, where mixtures of antagonist bacteria/phages will no doubt be needed to deal with such diversity. Phage technologies seem especially promising in contained facilities such as those used in flower and ornamental production, with field trials currently in progress. Research on the soil and rhizosphere microbiomes is increasing, which may open new opportunities for suppression and biocontrol. However, we need to understand the biology of these other soil microbes in much more detail, especially those present around growing roots and tubers, in order to determine any role they may have in controlling disease. As with all control options we must also examine carefully at any potential risks inherent in the use of such biocontrol agents (Sect. 2.5.1).

\subsubsection{Integrate Pest Management (IPM)}

Despite the establishment of sanitation measures and the huge efforts that have gone in to detection and discard of contaminated seeds and plant material, diseases caused by SRP continue to cause devastation in many countries (Chap. 7) and remain a major problem in potato production (Chap. 8). We know considerably more about the biology of these pathogens than we did when standard certification and sanitation methods began, yet an effective treatment that improves on or can work together with these methods remains elusive. Recent research advances, especially with the advent of genomics, have been considerable. Scientists from across the world are working together on this problem more than ever before, and we hope and expect that we will see the rewards of our work in the development of new control options in the near future. However, it is unlikely that there will be a 'silver bullet' for SRP disease control any time soon. Instead, a combination of methods as part of an integrated pest management (IPM) approach is our most likely route to success. 